\title{
Excited-state phase transition and onset of chaos in quantum optical models
}

\author{
P. Pérez-Fernández, ${ }^{1}$ A. Relaño, ${ }^{2}$ J. M. Arias, ${ }^{1}$ P. Cejnar, ${ }^{3}$ J. Dukelsky, ${ }^{4}$ and J. E. García-Ramos ${ }^{5}$ \\ ${ }^{1}$ Departamento de Física Atómica, Molecular y Nuclear, Facultad de Física, Universidad de Sevilla, Apartado 1065, E-41080 Sevilla, Spain \\ ${ }^{2}$ Grupo de Física Nuclear, Departamento de Física Atómica, Molecular y Nuclear, Universidad Complutense de Madrid, Avenida \\ Complutense s/n, E-28040 Madrid, Spain \\ ${ }^{3}$ Institute of Particle and Nuclear Physics, Faculty of Mathematics and Physics, Charles University, V Holešovičkách 2, Prague 18000, \\ Czech Republic \\ ${ }^{4}$ Instituto de Estructura de la Materia, CSIC, Serrano 123, E-28006 Madrid, Spain \\ ${ }^{5}$ Departamento de Física Aplicada, Universidad de Huelva, E-21071 Huelva, Spain
}

(Received 14 September 2010; revised manuscript received 16 December 2010; published 15 April 2011)

\begin{abstract}
We study the critical behavior of excited states and its relation to order and chaos in the Jaynes-Cummings and Dicke models of quantum optics. We show that both models exhibit a chain of excited-state quantum phase transitions demarcating the upper edge of the superradiant phase. For the Dicke model, the signatures of criticality in excited states are blurred by the onset of quantum chaos. We show that the emergence of quantum chaos is caused by the precursors of the excited-state quantum phase transition.
\end{abstract}

DOI: 10.1103/PhysRevE.83.046208

PACS number(s): 05.45.Mt, 05.30.Rt, 05.70.Fh, 42.50.Nn

\section{INTRODUCTION}

One of the goals of many-body physics is the understanding of quantum critical phenomena [1]. A quantum phase transition (QPT) appears in systems with Hamiltonian $H(\lambda)$, which show a sudden change of the ground-state properties when the control parameter $\lambda$ varies across a critical value $\lambda_{c}$. In the last few years, the connection of a QPT with the entanglement [2-4] and with the emergence of chaotic behavior $[2,4,5]$ has been investigated, motivated by the important role they could play in the current research related to quantum information technologies [6].

Very recently, a quantum critical phenomenon of a new type- the one related to excited states rather than to the ground state- has been discussed for several model systems [7-10]. An excited-state quantum phase transition (ESQPT) represents a nonanalytic evolution of individual excited states with the control parameter. It can also be observed as a singular variation of the state density with energy and entails dramatic dynamical consequences [11,12].

As ESQPTs have so far been identified in simple, mostly integrable systems, two natural questions arise: First, are the ESQPTs also generic in more complex, prevailingly chaotic systems? Second, how does the collapse of quantum energy levels, a typical ESQPT signature, affect the level repulsion, which is inherent in quantum chaos? The aim of this paper is to address these questions by analyzing a simplified model of collective interactions of matter and light, known as the Dicke model [13]. The Dicke model is a nonintegrable model, however, in the rotating wave approximation, it reduces to the integrable Jaynes-Cummings model [14]. Both versions have recently stirred up great interest, since the implementation of a tunable matter-light coupling represents a route to study quantum critical effects [15-17]. The QPT to a superradiant phase within the Dicke model was studied theoretically [2,18-20], and recently realized experimentally using a superfluid gas in an optical cavity [17].

This paper is organized as follows. In Sec. II we describe the models. In Sec. III we analyze the quantum phase transitions in both models, particulary those related to excited states. In
Sec. IV we study the relationship between the excited-state quantum phase transitions and the emergence of chaos in the Dicke model. Finally, in Sec. V we summarize the main conclusions.

\section{THE MODELS}

Both the Dicke and Jaynes-Cummings models assume a set of two-level atoms interacting by a dipole coupling of strength $\lambda$ with a single-mode bosonic field (cavity photons). The models are expressed via the creation and annihilation operators $b^{\dagger}$ and $b$, describing a bosonic mode with frequency $\omega$ (with $N_{b}=b^{\dagger} b$ being the number of photons), and the $S U(2)$ generators $\left\{J_{ \pm}, J_{z}\right\}$ describing the ensemble of $N_{a}$ two-level atoms with the level splitting $\omega_{0}$ in terms of a pseudospin of length $J=N_{a} / 2$. A useful realization of the $S U(2)$ algebra can be built through an array of spin- $\frac{1}{2}$ particles located on $2 J$ sites,

$$
J_{+}=\sum_{i=1}^{2 J} a_{\uparrow i}^{\dagger} a_{\downarrow i}=J_{-}^{\dagger}, \quad J_{z}=\frac{1}{2} \sum_{i=1}^{2 J}\left(a_{\uparrow i}^{\dagger} a_{\uparrow i}-a_{\downarrow i}^{\dagger} a_{\downarrow i}\right),
$$

where $a_{\uparrow i}^{\dagger}$ or $a_{\uparrow i}\left(a_{\downarrow i}^{\dagger}\right.$ or $\left.a_{\downarrow i}\right)$ create or annihilate spin-up (spindown) states of the fermion on site $i$ (the upper and lower state of the $i$ th atom), and the ladder operators $J_{ \pm}$describe collective spin flips along the array. $N_{\uparrow} \equiv J_{z}+J$ counts the number of atoms excited to the upper level. The system has two degrees of freedom associated with, e.g., $N_{b}$ and $J_{z}$.

The Jaynes-Cummings Hamiltonian [14]

$$
H_{1}(\lambda)=\omega_{0} J_{z}+\omega b^{\dagger} b+\frac{\lambda}{\sqrt{4 J}}\left[b J_{+}+b^{\dagger} J_{-}\right]
$$

conserves the quantity $M / 2=N_{b}+N_{\uparrow}$, which together with the Hamiltonian are the two required conserved quantities (integrals of motion) to assure the integrability of the system. We work with a fixed value, $M / 2=N_{a}$, which implies a finite dimension of the Hilbert space, and set $\omega>\omega_{0}$. 
The Dicke Hamiltonian [13]

$$
H_{2}(\lambda)=\omega_{0} J_{z}+\omega b^{\dagger} b+\frac{\lambda}{\sqrt{4 J}}\left[\left(b+b^{\dagger}\right)\left(J_{+}+J_{-}\right)\right]
$$

conserves the parity $\Pi=(-1)^{M / 2}$, but not the number $M$ itself. Therefore, it is not integrable. The Hilbert-space dimension for any $N_{a}$ is infinite, since states with unlimited photon numbers $N_{b}$ are coupled by the interaction term. From a practical point of view, this means that the photon space needs to be truncated by a certain maximal value $N_{b}^{\max }$. In the numerical calculations presented below, we checked that an increase of $N_{b}^{\max }$ does not cause noticeable changes in results. In these calculations, we set $\omega=\omega_{0}$, which corresponds to the resonance absorption and emission of photons by the atoms.

\section{QUANTUM PHASE TRANSITIONS}

These models serve as toy examples of the maser phase transition. Indeed, in the thermodynamic limit, $J \rightarrow \infty$, both Hamiltonians yield a QPT of the second order [2] in which properties are obtained from a relevant semiclassical analysis [12]. The critical values of the coupling strength are $\lambda_{c}=\sqrt{\left(\omega_{0}-\omega\right)^{2} / 2}$ for the Jaynes-Cummings model ${ }^{1}$ and $\lambda_{c}=\sqrt{\omega \omega_{0} / 2}$ for the the Dicke model. Below the critical point, a normal phase exists, in which the ground state is similar to that at $\lambda=0$, given by the photon vacuum $\left(N_{b}=0\right)$ combined with a maximally excited $\left(J_{z}=+J\right)$ or a totally unexcited $\left(J_{z}=-J\right)$ state of the atom array. The first case is valid for the Jaynes-Cummings model with $\omega>\omega_{0}$ and $M=4 J$, and the second one is valid for the Dicke model. For a coupling strength below $\lambda_{c}$ in the thermodynamic limit, the $\lambda=0$ ground-state form is preserved with expectation values $\left\langle N_{b}\right\rangle=0,\left\langle J_{z}\right\rangle= \pm J$, and ground-state energy $E_{0}^{\text {norm }}=$ $\pm J \omega_{0}$. When crossing the critical value $\lambda_{c}$, the ground state eventually flips to a form with expectation values $\left\langle N_{b}\right\rangle>0$ and $-J<\left\langle J_{z}\right\rangle<+J$, and decreasing energy $E_{0}(\lambda)<E_{0}^{\text {norm }}$, in which both the photon field and the atomic array acquire partial, macroscopic excitations. This regime can be interpreted as a superradiant phase [2,18]. The quantities $\left\langle J_{z}\right\rangle$ (or $\left\langle N_{\uparrow}\right\rangle$ ) and $\left\langle N_{b}\right\rangle$ represent suitable order parameters to characterize the superradiant phase transition.

The ground-state QPT in both models is followed for $\lambda>$ $\lambda_{c}$ by a chain of excited-state phase transitions located at the critical energy, coinciding approximately with the ground-state energy of the normal phase, $E_{c}=E_{0}^{\text {norm }}$. A detailed discussion of the ESQPT effects and their semiclassical roots is given elsewhere [12]. The behavior of the order parameter $\left\langle J_{z}\right\rangle$ close to the critical energy $E_{c}$ is given by

$$
\left\langle J_{z}\right\rangle=\left\langle J_{z_{c}}\right\rangle+A\left|E-E_{c}\right|^{\alpha},
$$

which is characterized by a critical exponent $\alpha$. Results for the Jaynes-Cummings model with 1000 atoms are plotted in Fig. 1 for $\lambda=1.5$. The red points correspond to the expectation value of $J_{z}$ in each eigenstate with eigenvalue $E$. The continuous

\footnotetext{
${ }^{1}$ Note that we are describing a transition with a fixed number of particles, therefore this critical value differs from that of Ref. [2]. For more details, see [12].
}

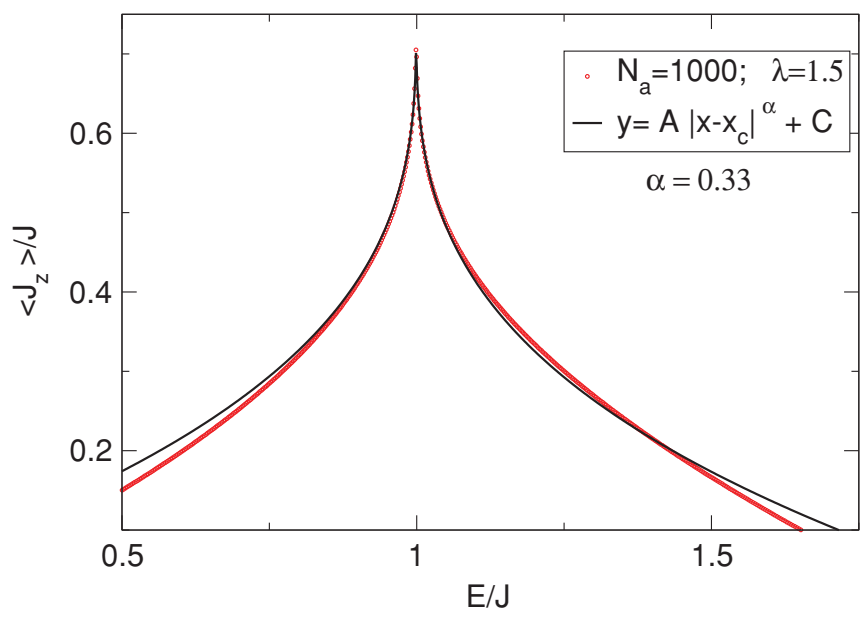

FIG. 1. (Color online) Scaled atomic inversion as a function of energy for the Jaynes-Cummings model, with calculations for $\omega_{0}=1$, $\omega=2, \lambda=1.5$, and $J=500$.

black curve shows a fit using Eq. (4) with the critical exponent $\alpha=0.33$. The cusp singularity at $E_{c} / J=1$ is characterized by the value $\left\langle J_{z c}\right\rangle=J$, hence $\left\langle N_{\uparrow}\right\rangle=N_{a}$ (not seen in the figure). This can be described as the $\lambda<\lambda_{c}$ ground-state structure propagating through the spectrum along the line $E=E_{c}$, where we indeed observe multiple avoided crossings of individual levels [12].

Similar results for the Dicke model with 60 atoms and $\lambda=1.5$ are plotted in Fig. 2 . The thin red oscillating line represents numerical data for $\left\langle J_{z}\right\rangle$ obtained as an average over 20 eigenstates around the eigenvalue $E$; the points have been joined by a line for easier visualization. The thick black curve corresponds to a fit by Eq. (4). The number of atoms is much smaller than that used in the previous calculation because of a rather large value of $N_{b}^{\max }$ needed to get convergence for the levels above the critical energy. In particular, results for $E / J>1$ (the flat part of the numerical dependence in Fig. 2) are not converged.

A comparison with Fig. 1 shows that the results for the Dicke model are fuzzier than those for the Jaynes-Cummings model. They exhibit sizable fluctuations around a smooth

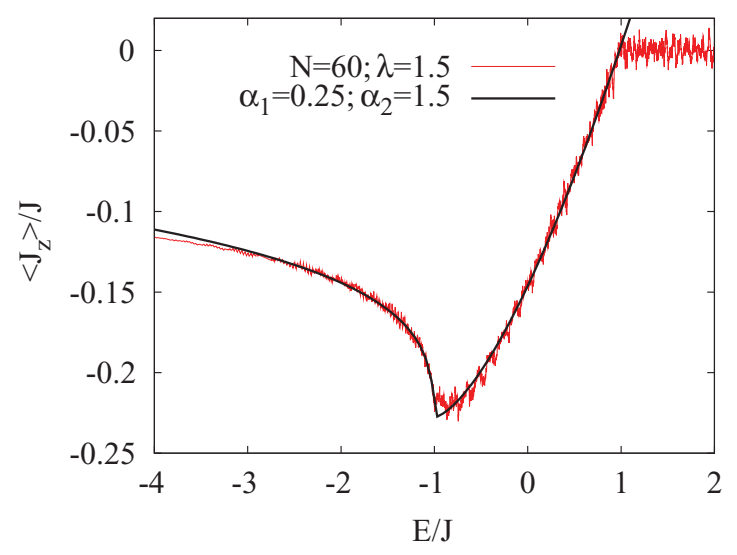

FIG. 2. (Color online) Scaled atomic inversion as a function of energy for the Dicke model, with calculations for $\omega_{0}=\omega=1, \lambda=$ $1.5, J=30$, and results smoothed over 20 points. 
dependence. Another peculiarity of the Dicke model is that the order parameter is bimodal with two critical exponents: $\alpha_{1}=$ 0.25 for $E<E_{c}$, and $\alpha_{2}=1.5$ for $E>E_{c}$. Despite these differences, Fig. 2 shows a qualitatively similar dependence as Fig. 1, demonstrating a related type of singularity in the Dicke model at the critical energy $E_{c} / J=-1$. We therefore conclude that for $\lambda>\lambda_{c}$, both models exhibit an ESQPT at $E_{c}=E_{0}^{\text {norm }}$. This critical energy terminates the domain of the superradiant phase present at low temperatures [18].

\section{CHAOS AND EXCITED-STATE QUANTUM PHASE TRANSITIONS}

We know that the Dicke model is nonintegrable and partly chaotic. The superradiant transition at zero temperature was shown [2] to be correlated with a crossover from ordered to chaotic behavior. Hence one may ask whether quantum chaos is also somehow related to the critical behavior of excited states. It can be anticipated that chaotic properties of the spectrum introduce large fluctuations that partly hide the singular dependence at the critical point in Fig. 2. However, chaos and ESQPTs have some properties which are difficult to conciliate. On one hand, the most significant feature of quantum chaos is the level repulsion, which entails a null probability of finding two levels at the same energy [21]. On the other hand, an ESQPT as a rapid restructuring of excited states is typically connected with a rather close approach of levels (numerous sharp avoided crossings), often with a singular accumulation of levels at $E=E_{c}$ [7-9,11]. Therefore, the relation between the level repulsion and spectral signatures of an ESQPT constitutes an interesting theoretical challenge.

Let us consider the Dicke Hamiltonian with a value of $\lambda$ above $\lambda_{c}$, where the system is partly chaotic [2]. To analyze how chaos and the ESQPT can dwell together, we calculate the spacing distribution $P(s)$, where $s$ is a normalized $(\bar{s}=1)$ distance between two neighboring levels, on both sides of the critical energy $E_{c}$. This distribution is known to interpolate between the Poissonian and Wigner forms, $P^{\mathrm{P}}=e^{-s}$ and $P^{\mathrm{W}}=\frac{\pi}{2} s e^{-\pi s^{2} / 4}$, respectively, as the system transforms from a regular to a chaotic regime [21]. In order to have a large number of levels below the critical energy, we present results for $\lambda=3$; different choices lead to similar pictures.

In Fig. 3 we show the $P(s)$ distributions calculated for both subcritical $\left(E<E_{c}\right)$ and supercritical $\left(E>E_{c}\right)$ parts of the spectrum, and for various atom numbers (see caption for details). In all cases, we used the same number of levels for building the histograms on both sides of the critical energy. For $E>E_{c}$, the spectral statistics closely follow the Wigner surmise. In contrast, for $E<E_{c}$, the shape of the histograms is not clear: while for the $N_{a}=46$ case, it is rather close to the Poissonian distribution, for lower atom numbers, it yields neither the Wigner nor the Poissonian form. The most relevant fact is that below $E_{c}$ we observe $P(s=0)>0$, while above $E_{c}$ we obtain $P(s=0) \approx 0$. In other words, the levels start to repel each other when crossing the ESQPT critical energy.

To obtain a more quantitative description, we analyze how the spectral statistics change as one moves in the spectrum. To increase the accuracy of the procedure, we rely on the accumulated spacing distribution $F(s)=\int_{0}^{s} d x P(x)$. Given a

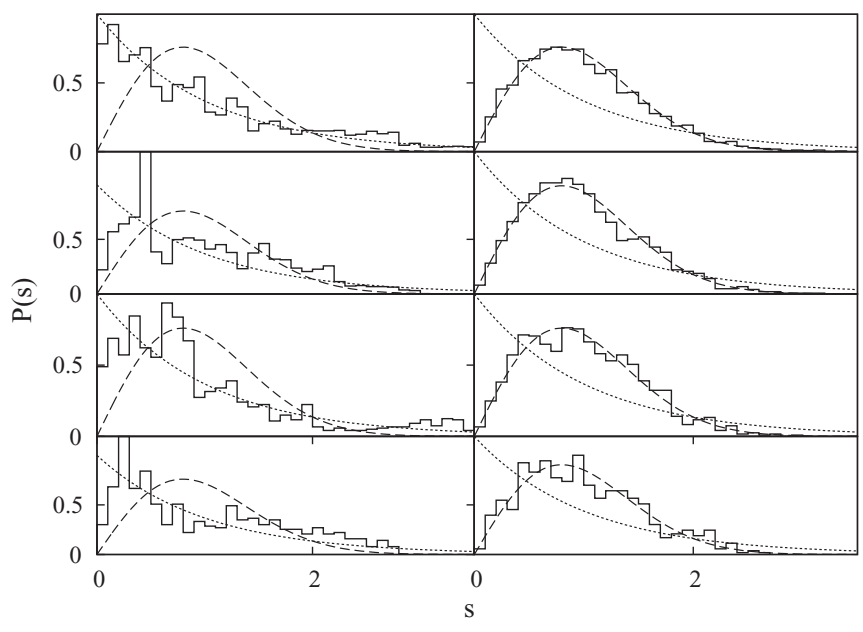

FIG. 3. Nearest-neighbor spacing distribution $P(s)$ for the Dicke model with $\lambda=3$. Histograms are shown for $E<E_{c}$ (left column) and $E>E_{c}$ (right column). The rows from bottom to top correspond to $N_{a}=22,30,38$, and 46 . The short dashed lines correspond to the Poisson distribution, and the long dashed lines correspond to the Wigner-Dyson distribution.

numerical sequence of spacings $\left\{s_{i}\right\}$, we measure its "distance" from the Wigner surmise by means of the following quantity:

$$
\Delta=\frac{\sum_{i}\left[F^{\mathrm{W}}\left(s_{i}\right)-F\left(s_{i}\right)\right]^{2}}{\sum_{i}\left[F^{\mathrm{W}}\left(s_{i}\right)-F^{\mathrm{P}}\left(s_{i}\right)\right]^{2}},
$$

where $F^{\mathrm{W}}$ and $F^{\mathrm{P}}$ are accumulated distributions derived from $P^{\mathrm{W}}$ and $P^{\mathrm{P}}$, respectively. Therefore, $\Delta=0$ if the numerical sequence follows the Wigner distribution, while $\Delta=1$ for a Poissonian sequence. We have constructed sequences of 200 consecutive spacings and, using Eq. (5), we have calculated the distance $\Delta$ of each sequence from the Wigner statistics. These distances are plotted in Fig. 4 as a function of the mean energy of the respective sequence. We can see that a quite abrupt transition from finite values of $\Delta$ to $\Delta \sim 0$ takes place just below the critical energy $E_{c}$. Although the range of the covered values of $N_{a}$ is not large enough to approach the thermodynamic limit, it suffices to confirm that the critical behavior in the order parameter is accompanied by a change in the spectral statistics. Furthermore, as this change is so abrupt in finite size systems, we are led to attribute it to the precursors of the ESQPT.

We are now able to go beyond the hypothesis of Ref. [2], stating that the spectrum of the Dicke model with $\lambda>\lambda_{c}$ is regular at low energies. From our numerical results, we conclude that a transition to chaos takes place around the critical energy $E_{c}$. A compact picture of various dynamical regimes implicit in the Dicke model can therefore be stated as follows: We start at $\lambda=0$, with the ground state having $N_{b}=$ $N_{\uparrow}=0$. Increasing $\lambda$ leaves the ground state unperturbed until we cross the critical point $\lambda_{c}$ for the superradiant transition, where the expectation values $\left\langle N_{b}\right\rangle$ and $\left\langle N_{\uparrow}\right\rangle$ start to increase. For any value of $\lambda>\lambda_{c}$, there exists a region above the ground state in which we find no level repulsion, $P(s=0)>0$. This seems to be a common feature of almost the entire superradiant domain in the $\lambda \times E$ phase diagram. If the energy is increased 


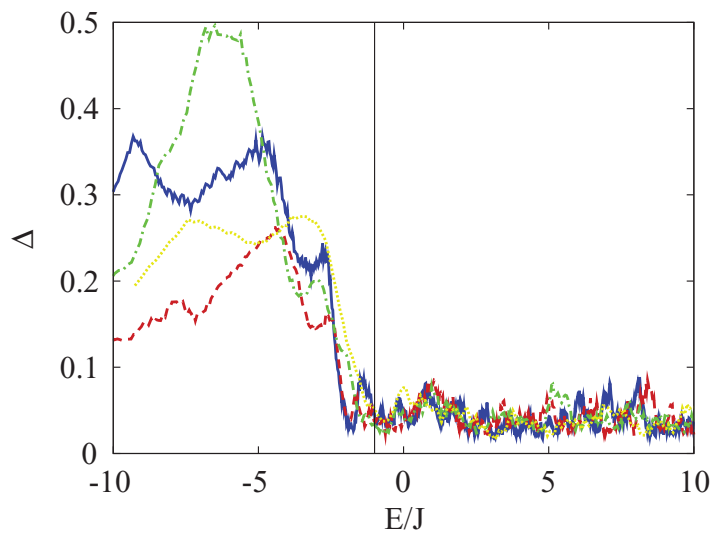

FIG. 4. (Color online) Onset of chaos in the Dicke model, with $\lambda=3$ measured by the distance (5) of actual level statistics from the Wigner distribution. The distance $(\Delta)$ is plotted as a function of the mean scaled energy of the relevant fraction of the spectrum, with the vertical line indicating the critical energy. Calculations are done for various atom numbers: $N_{a}=22$ (very light yellow dotted line), 30 (light green dashed-dotted line), 38 (dark red dashed line), and 46 (very dark blue solid line).

closely below the critical value $E_{c}=-J \omega_{0}$, where $\left\langle N_{\uparrow}\right\rangle$ drops sharply, the level repulsion sets in, leading the spectrum to the Wigner type of statistics. Above $E_{c}$, the system becomes fully chaotic.

\section{CONCLUSIONS}

In conclusion, we have demonstrated the existence of an excited-state quantum phase transition in two models describing the collective matter-light interaction. In the integrable Jaynes-Cummings model, the ESQPT leads to a neat nonanalyticity of the order parameter $\left\langle J_{z}\right\rangle$ at the critical energy $E_{c}$. The nonintegrable Dicke model exhibits a similar type of ESQPT, but the signatures are blurred by the onset of chaotic behavior in the spectrum. Level repulsion (a fundamental feature of quantum chaotic systems) and a cumulation of sharp avoided crossings (a typical signature of an ESQPT) are difficult to conciliate in general. However, our numerical calculations show that a crossover, from the regime with no level repulsion to the one with the Wigner level statistics, takes place precisely around the critical energy. Moreover, our results are compatible with the hypothesis that this abrupt emergence of level repulsion is caused by the precursors of the ESQPT, in a similar way as discussed in Ref. [2] for the ground state. We anticipate the existence of a similar qualitative behavior in other nonintegrable systems with ESQPTs. Moreover, other dynamical effects of ESQPTs, such as, for instance, anomalous decoherence factors previously obtained in the Lipkin model [11], are expected to take place in a fuzzier manner in quantum chaotic systems.

\section{ACKNOWLEDGMENTS}

This work was supported by the Czech Science Foundation (Grant No. 202/09/0084), Czech Ministry of Education (Grant No. MSM 0021620859), Spanish Ministerio de Ciencia e Innovación and European regional development fund (Grants No. FIS2009-07277, No. FIS2008-04189, No. FIS200911621-C02-01, and No. FPA2007-63074), CPAN-Ingenio (Grant No. CSPD-2007-00042-Ingenio 2010), and Junta de Andalucía (Grants No. FQM160, No. FQM318, No. P05FQM437, and No. P07-FQM-02962). The work of P. P.-F. is funded by a FPU grant of the Spanish Ministerio de Educación, and the work of A. R. is funded by the program CPAN Consolider-Ingenio 2010.
[1] S. Sachdev, Quantum Phase Transitions (Cambridge University Press, Cambridge, 1999).

[2] C. Emary and T. Brandes, Phys. Rev. Lett. 90, 044101 (2003); Phys. Rev. E 67, 066203 (2003); N. Lambert, C. Emary, and T. Brandes, Phys. Rev. Lett. 92, 073602 (2004).

[3] L. Amico, R. Fazio, A. Osterloh, and V. Vedral, Rev. Mod. Phys. 80, 517 (2008).

[4] E. A. Chagas and K. Furuya, Phys. Lett. A 372, 5564 (2008).

[5] T. Deguchi and P. K. Ghosh, Phys. Rev. E 80, 021107 (2009); T. Deguchi, P. K. Ghosh, and K. Kudo, Phys. Rev. E 80, 026213 (2009).

[6] Quantum Computers, Algorithms and Chaos, edited by G. Casati, D. L. Shepelyansky, P. Zoller, and G. Benenti, International School of Physics "Enrico Fermi," Vol. 162 (IOS, Amsterdam, 2006).

[7] P. Cejnar, M. Macek, S. Heinze, J. Jolie, and J. Dobeš, J. Phys. A 39, L515 (2006).

[8] M. A. Caprio, P. Cejnar, and F. Iachello, Ann. Phys. (NY) 323, 1106 (2008).

[9] P. Cejnar and P. Stránský, Phys. Rev. E 78, 031130 (2008).

[10] P. Ribeiro, J. Vidal, and R. Mosseri, Phys. Rev. Lett. 99, 050402 (2007); Phys. Rev. E 78, 021106 (2008).
[11] A. Relaño, J. M. Arias, J. Dukelsky, J. E. García-Ramos, and P. Pérez-Fernández, Phys. Rev. A 78, 060102 (2008); P. PérezFernández, A. Relaño, J. M. Arias, J. Dukelsky, and J. E. GarcíaRamos, Phys. Rev. A 80, 032111 (2009).

[12] P. Pérez-Fernández, P. Cejnar, J. M. Arias, J. Dukelsky, J. E. García-Ramos, and A. Relaño, Phys. Rev. A 83, 033802 (2011).

[13] R. H. Dicke, Phys. Rev. 93, 99 (1954).

[14] E. T. Jaynes and F. W. Cummings, Proc. IEEE 51, 89 (1963); M. Tavis and F. W. Cummings, Phys. Rev. 170, 379 (1968).

[15] F. Illuminati, Nature Phys. 2, 803 (2006).

[16] A. D. Greentree, C. Tahan, J. H. Cole, and L. C. L. Hollenberg, Nature Phys. 2, 856 (2006).

[17] K. Baumann, C. Guerlin, F. Brennecke, and T. Esslinger, Nature (London) 464, 1301 (2010).

[18] K. Hepp and E. H. Lieb, Ann. Phys. (NY) 76, 360 (1973).

[19] D. Nagy, G. Kónya, G. Szirmai, and P. Domokos, Phys. Rev. Lett. 104, 130401 (2010).

[20] B. M. Rodríguez-Lara and Ray-Kuang Lee, J. Opt. Soc. Am. B 27, 2443 (2010).

[21] H.-J. Stöckmann, Quantum Chaos: An Introduction (Cambridge University Press, Cambridge, 1999). 